\title{
A study on comparison between fuzzy assignment problems using trapezoidal fuzzy numbers with average method
}

\author{
S. Manimaran ${ }^{1}$ and M. Ananthanarayanan ${ }^{2}$ \\ ${ }^{1}$ Department of Mathematics, R.K.M. Vivekananda College, Chennai-600 005, India \\ ${ }^{2}$ Department of Mathematics, A.M. Jain College, Chennai-600 114,India \\ ananthanarayanan47@yahoo.com
}

\begin{abstract}
Assignment problem is very often used in solving problems of engineering and management science. In this paper, trapezoidal fuzzy numbers are considered which are more realistic and general in nature. The fuzzy assignment problem has been transformed into crisp assignment problem in the LPP form solved by using LINGO 9.0 and the results were compared with average method.

Subject classification: Fuzzy Logic- 03B52
\end{abstract}

Keywords. Fuzzy sets, fuzzy numbers, fuzzy assignment problem , fuzzy ranking, hungerian method.

Introduction

Assignment problem (AP) is used worldwide in solving real world problems. An assignment problem plays an important role in industry and other applications. In an assignment problem, $\mathrm{n}$ jobs are to be performed by $\mathrm{n}$ persons depending on their efficiency to do the job. In this problem $C^{i j}$ denotes the cost of assigning the $j^{\text {th }}$ job to the $i^{\text {th }}$ person. We assume that one person can be assigned exactly one job; also each person can do at most one job. This Problem is to find an optimal assignment so that the total cost of performing all jobs is minimum or the total profit is maximum.

In this paper we investigate a more realistic problem, namely the assignment problem with fuzzy cost or times $\overline{c_{i j}}$. Since the objectives are to minimize the total cost or to maximize the total profit, subject to some crisp constrains, the objective values of the objective function by some ranking method for fuzzy numbers is to find the best alternative. On the basis of this idea the Yager's ranking method (Yager, 1981) has been adopted to transform the fuzzy assignment problem to a crisp one so that the conventional solution may be applied to solve the AP.

The idea is to transform a problem with fuzzy parameters to a crisp version in the LPP form and to solve it by the simplex method .Other than the fuzzy assignment other applications of this method can be tried in project scheduling, maximal flow, transportation problem etc. Fuzzy assignment problem and fuzzy ranking method

Lin and Wen (2004) solved the assignment problem with fuzzy interval number costs by a labeling algorithm. Sakawa et al. (2001) dealt with actual problems on production and work force assignment in a housing material manufacturer and a sub contract firm and formulated two kinds of two-level programming problems. Applying the interactive fuzzy programming for two-level linear and linear fractional programming problems, they derived satisfactory solution to the problems and compared the results. They examined actual planning of

Research article

(C) Indian Society for Education and Environment (iSee)

"Fuzzy sets"

http://www.indjst.org the production and the work force assignment of the two firms to be implemented. Chen (1985) proved some theorems and proposed a fuzzy assignment model that considers all individuals to have the same skills. Wang (1987) solved a similar model by graph theory. Dubois and Fortempts (1999) surveyed refinements of the ordering of solutions supplied by the max-min formulation, namely the duiscriming partial ordering and the leximin complete preordering. They have given a general algorithm which computes all maximal solutions in the sense of these relations. Different kinds of fuzzy transportation problems were solved earlier (Chanas et al., 1984; Igeartaigh, 1984; Chen ,1985; Chanas \& Kuchta,1996; Chanas \& Kuchta,1998).

Dominance of fuzzy numbers can be explained by many ranking methods. Yager's ranking method (Yager, 1981 ) is a robust ranking technique which satisfies the properties of compensation, linearity and additive. We have applied Yager's ranking technique in this paper.

Zadeh first introduced fuzzy set as a mathematical way of representing impreciseness of vagueness in everyday life. Fuzzy set is characterized by a membership function mapping the elements of a domain, space, or universe of discourse $X$ to the unit interval $[0,1]$ (Zadeh, 1965).

Fuzzy set $A$ in a universe of discourse $X$ is defined as the following of pairs;

$$
A=\left\{\left(x, \mu_{A}(x): x \in X\right\} \text {. Here } \mu_{A}: X \longrightarrow[0,1]\right. \text { is a }
$$
mapping called membership function of the fuzzy set $\mathrm{A}$ and $\mu_{A}(x)$ is a called membership value or degree of membership of $x \in X$ in the fuzzy set $\mathrm{A}$. Larger value of $\mu_{A}(x)$, stronger is the grade of membership form in $\mathrm{A}$. These membership grades are often represented by real numbers ranging from a minimum of 0 to a maximum of 1

Normal fuzzy set: A fuzzy set $A$ of the universe of discourse $X$ is called a normal fuzzy set implying that there exist at least one $x \in X$ such that $\mu_{A}(x)=1$. 
Convex fuzzy set: The fuzzy set $A$ is convex if and only if ,for any $x_{1}, x_{2} \in X$, the membership function of $\mathrm{A}$ satisfy the inequality

$\mu_{A}\left\{\lambda x_{1}+(1-\lambda) x_{2}\right\} \geq \min \left\{\mu_{A}\left(x_{1}\right), \mu_{A}\left(x_{2}\right)\right\}, 0 \leq \lambda \leq 1$

For a Trapezoidal fuzzy number $\mathrm{A}(\mathrm{x})$, it can be represented by $\mathrm{A}(\mathrm{a}, \mathrm{b}, \mathrm{c}, \mathrm{d} ; 1)$ with the membership function $\mu(x)$ is given by

$\mu(x)=\left\{\begin{array}{l}\frac{(x-a)}{(b-a)}, a \leq x \leq b \\ 1, b \leq x \leq c \\ \frac{(d-x)}{(d-c)}, c \leq x \leq d \\ 0, \text { otherwise }\end{array}\right.$

Satisfying the following conditions:

(1) $\mu(x)$ is a continuous mapping from $\mathrm{R}$ to the closed interval[ $[0,1]$

(2) $\mu(x)=0$ for all $x \in(-\infty, a)$;

(3) Strictly increasing and continuous on $[a, b]$

(4) $\mu(x)=1$ for all $x \in[c, d]$;

(5) Strictly decreasing and continuous on [c,d]

(6) $\mu(x)=0$ for all $x \in[d, \infty)$;

The graphic representation of a trapezoidal fuzzy number is shown in Fig.1

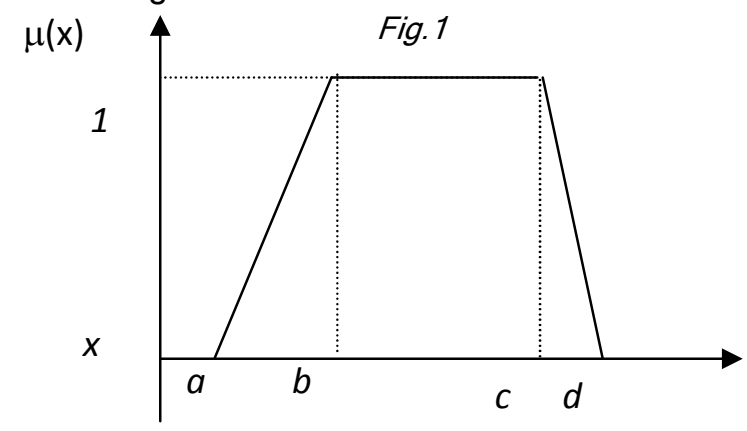

The Proposed method

The assignment problem can be stated in the form of nxn cost matrix $\left[\mathrm{C}_{\mathrm{ij}}\right]$ of real numbers

Mathematically assignment problem can be stated as

$$
\operatorname{Minimize}(z)=\sum_{i=1}^{n} \sum_{j=1}^{n} c_{i j} x_{i j}, i=1,2 \ldots n, j=1,2 \ldots n
$$

Subject to

$x_{i j} \in\{0,1\}$, where

$x_{i j}=\left\{\begin{array}{l}1, \text { ifthe }^{\text {th }} \text { personassigned } j^{\text {th }} \text { job } \\ 0, \text { otherwise }\end{array}\right.$
Vol. 5 No. 4 (Apr 2012)

ISSN: 0974- 6846

is the decision variable denoting the assignment of the person I to job j. Cij is the cost of assigning the

$j^{\text {th }}$ job to the $i^{\text {th }}$ person. The objective is to minimize the total cost of assigning all the jobs to the available persons.(one job to one person) When the costs or time

$\overline{c_{i j}}$ are fuzzy numbers ,then the total cost becomes a fuzzy number.

$\bar{z}=\sum_{i=1}^{n} \sum_{j=1}^{n} \bar{c}_{i j} x_{i j} \quad$ Hence it cannot be minimized directly.

For solving the problem we defuzzify the fuzzy cost coefficients into crisp ones by a fuzzy number ranking method.

Yager's ranking technique[11] is a robust ranking technique which satisfies compensation ,linearity, and additive properties and provides results which are consistent with human intuition. Given a convex fuzzy number $\bar{c}$,the Yager's Ranking index is defined by $Y(\bar{C})=\int_{0}^{1} 0.5\left(c_{\alpha}^{L}+c_{\alpha}^{U}\right) d \alpha$, where $\left(c_{\alpha}^{L}, c_{\alpha}^{U}\right)$ is the $\alpha$ level cut of the fuzzy number $\overline{c_{i j}}$

In this paper we use this method for ranking the objective values. The Yager's ranking index $\mathrm{Y}\left(\overline{c_{i j}}\right)$ gives the representative value of the fuzzy number $\bar{c}$. It satisfies the linear and additive property:

If $\bar{A}=a \bar{B}+b \bar{c}$ and $\bar{D}=k \bar{E}-t \bar{F}$, where $\mathrm{a}, \mathrm{b}, \mathrm{k}, \mathrm{t}$ are constants, then we have

$$
Y(\bar{A})=a Y(\bar{B})+b Y(\bar{c}) \quad \text { and }
$$

$\mathrm{Y}(Y(\bar{D})=k Y(\bar{E})-t Y(\bar{F})$. On the basis of this property and fuzzy assignment problem can be transformed in to a crisp assignment problem in the LPP form. The ranking technique of Yager is:

$$
\text { If } Y(\bar{U}) \leq Y(\bar{V}) \text {, then } \bar{U} \leq \bar{V} \text { i.e. } \min (\bar{U}, \bar{V})=\bar{U}
$$

For the assignment problem (1) with fuzzy objective function

$\operatorname{Minimize}(\bar{z})=\sum_{i=1}^{n} \sum_{j=1}^{n} \bar{c}_{i j} x_{i j}$, we apply yager's ranking method [11] (using linearity and additive property) to get the minimum objective value $\bar{z}^{*}$ from the formulation

$\mathrm{Y}\left(\bar{Z}^{*}\right)=$

$\operatorname{Minimize}(z)=\sum_{i=1}^{n} \sum_{j=1}^{n} c_{i j} x_{i j}, i=1,2 \ldots n, j=1,2 \ldots n$

Subject to 
$\sum_{j=1}^{n} x_{i j}=1, i=1,2, \ldots . n$

$\sum_{i=1}^{n} x_{i j}=1, j=1,2, \ldots n$

$x_{i j} \in\{0,1\}$, where

$x_{i j}=\left\{\begin{array}{l}1, \text { ifthe }^{\text {th }} \text { personassigned } j^{\text {th }} \text { job } \\ 0, \text { otherwise }\end{array}\right.$

is the decision variable denoting the assignment of the person I to job $\mathrm{j} . \overline{c_{i j}}$ is the cost of assigning the jth job to the $i$ th person. The objective is to minimize the total cost of assigning all the jobs to the available persons (one job to one person).

Since $\mathrm{Y}\left(\overline{c_{i j}}\right)$ are crisp values, this problem (2) is obliviously the crisp assignment problem of the form (1) which can be solved by conventional method .Once the optimal solution $x^{*}$ of model (2) is found,the optimal fuzzy objective value $\bar{z}^{*}$ of the original problem can be calculated as

$\bar{z}^{*}=\sum_{i=1}^{n} \sum_{j=1}^{n} \bar{c}_{i j} x_{i j}^{*}$,

\section{Numerical example}

Let us consider a fuzzy Assignment Problem with rows representing 4 persons $A, B, C, D$ and columns representing 4 jobs ob1,job2,job3,job4, The cost matrix $\left[\overline{c_{i j}}\right]$ is given whose elements are trapezoidal fuzzy numbers. The problem is to find the optimal assignment so that the total cost of job assignment becomes minimum

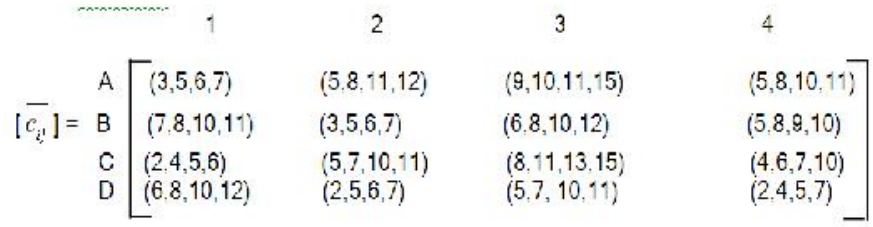

Solution: In conformation to Model (2) the fuzzy assignment problem can be formulated in the following mathematical programming form:

Min $\quad\left[\quad Y(\quad 3,5,6,7) x_{11}+Y(5,8,11,12)\right.$

$+Y(9,10,11,15) x_{13}+Y(5,8,10,11) x_{14}$

$+\mathrm{Y}(7,8,10,11) x_{21}+\mathrm{Y}(3,5,6,7) \quad x_{22}+\mathrm{Y}(6,8,10,12) \quad x_{23}$

$+Y(5,8,9,10) x_{24}$

$+Y(2,4,5,6) x_{31}+Y(5,7,10,11) \quad x_{32}+Y(8,11,13,15) \quad x_{33}$

$+Y(4,6,7,10) x_{34}$

$$
\begin{aligned}
& +Y(6,8,10,12) x_{41}+\mathrm{Y}(2,5,6,7) x_{42}+\mathrm{Y}(5,7,10,11) x_{43} \\
& \left.+\mathrm{Y}(2,4,5,7) x_{44}\right]
\end{aligned}
$$

$$
\begin{array}{lr}
\text { S.t } x_{11}+x_{12}+x_{13}+x_{14}=1, & x_{11}+x_{21}+x_{31}+x_{41}=1, \\
x_{21}+x_{22}+x_{23}+x_{24}=1, & x_{12}+x_{22}+x_{32}+x_{42}=1, \\
x_{31}+x_{32}+x_{33}+x_{34}=1, & x_{13}+x_{23}+x_{33}+x_{43}=1,
\end{array}
$$

$x_{41}+x_{42}+x_{43}+x_{44}=1, \quad x_{14}+x_{24}+x_{34}+x_{44}=1$,

Now we calculate $Y(3,5,6,7)$ by applying the Yager's ranking method. The membership function of the trapezoidal number $(3,5,6,7)$

$\mu(x)=\left\{\begin{array}{l}\frac{(x-3)}{2}, 3 \leq x \leq 5 \\ 1,5 \leq x \leq 6 \\ \frac{(7-x)}{1}, 6 \leq x \leq 7 \\ 0, \text { otherwise }\end{array}\right.$

The $\alpha$-cut of the fuzzy number $(3,5,6,7)$ is $\left(c_{\alpha}^{L}, c_{\alpha}^{U}\right)=(2 \alpha+3,7-\alpha)$ for which

$$
\begin{aligned}
& \mathrm{Y}\left(\overline{C_{11}}\right)=\mathrm{Y}(3,5,6,7)=\int_{0}^{1} 0.5\left(c_{\alpha}^{L}+c_{\alpha}^{U}\right) d \alpha= \\
& \int_{0}^{1} 0.5(2 \alpha+3+7-\alpha) d \alpha=\int_{0}^{1} 0.5(\alpha+10) d \alpha=5.25
\end{aligned}
$$

Proceeding similarly, the Yager's ranking indices for the fuzzy costs $\overline{c_{i j}}$ are calculated as:

$\mathrm{Y}\left(\overline{c_{12}}\right)=9, \quad \mathrm{Y}\left(\overline{c_{13}}\right)=11.25, \quad \mathrm{Y}\left(\overline{c_{14}}\right)=8, \quad \mathrm{Y}\left(\overline{c_{21}}\right)=9$, $\mathrm{Y}\left(\overline{c_{22}}\right)=5.25, \quad \mathrm{Y}\left(\overline{c_{23}}\right)=9, \quad \mathrm{Y}\left(\overline{c_{24}}\right)=8, \mathrm{Y}\left(\overline{c_{31}}\right)=4.25$, $\mathrm{Y}\left(\overline{c_{32}}\right)=8.25 \mathrm{Y}\left(\overline{c_{33}}\right)=11.75, \quad \mathrm{Y}\left(\overline{c_{34}}\right)=6.75, \quad \mathrm{Y}\left(\overline{c_{41}}\right)=9$, $\mathrm{Y}\left(\overline{c_{42}}\right)=5, \quad \mathrm{Y}\left(\overline{c_{43}}\right)=8.25, \mathrm{Y}\left(\overline{c_{44}}\right)=4.5$

We replace these values for their corresponding $\overline{c_{i j}}$ in (3)which results in a conventional assignment problem in the L.P.P form. We solve it by using Lingo 9.0 to get the following optimal solution

$x_{13}^{*}=x_{22}^{*}=x_{31}^{*}=x_{44}^{*}=1$,

$x_{11}^{*}=x_{12}^{*}=x_{14}^{*}=x_{21}^{*}=x_{23}^{*}=x_{24}^{*}=x_{32}^{*}=x_{33}^{*}=x_{34}^{*}=x_{41}^{*}=x_{42}^{*}=x_{43}^{*}$

$=0$ 'with the optimal objective value $\mathrm{Y}\left(\overline{z^{*}}\right)=25.25$ which represents the optimal total cost. In other words the optimal assignment is

$A \rightarrow 3, B \rightarrow 2, c \rightarrow 1, D \rightarrow 4$.

The fuzzy optimal total cost is calculated as 
$\overline{c_{13}}+\overline{c_{22}}+\overline{c_{31}}+\overline{c_{44}}=$

$(9,10,11,15)+(3,5,6,7)+(2,4,5,6)+(2,4,5,7)$

$(16,23,27,35)$

Also we find that $Y\left(\overline{z^{*}}\right)=Y(16,23,27,35)=25.25$.

Average Method

Description of the Model: Find the component wise average of the trapezoidal fuzzy numbers forms an assignment problem

Conclusion

Average method is proposed to solve the fuzzy assignment problems with trapezoidal fuzzy numbers in real life situations. To illustrate the proposed method a numerical example is solved and obtained the results are explained by comparing with the average method. If there is no uncertainty about the cost then the average method gives the same results as in the fuzzy assignment problem with trapezoidal fuzzy numbers.

\section{References}

1. Chanas S and Kuchta D (1996) A concept of the optimal solution of the transportation problem with fuzzy cost coefficients. Fuzzy Sets \& System. 82, 299305.

2. Chanas S and Kuchta D (1998) Fuzzy integer transportation problem. Fuzzy Sets \& System. 98, 291-298.

3. Chanas S, Kolodziejczyk W and Machaj A (1984) A fuzzy approach to the transportation problem. Fuzzy Sets \& System. 13, 211-221.

4. Chen CB and Klein CM (1997) A simple approach to ranking a group of aggregated fuzzy utilities IEEE Trans Syst., Man, Cybern. B. 27, 26-35.

5. Chen MS (1985) On a fuzzy assignment problem. Tamkang J 22, 407-411.

6. Chi-Jen Lin and Ue-Pyng Wen (2004) A labeling algorithm for the fuzzy assignment problem. Fuzzy Sets. Sys. 142, 373-391.

7. Dubois $D$ and Fortemps $P$ (1999) Computing improved optimal solutions to max-min flexible constraint satisfaction problems. Euro. J. Oper. Res. 118, 95126.

8. Fortemps $P$ and Roubens M (1996) Ranking and defuzzification methods based on area compensation. Fuzzy Sets \& System. 82, 319-330.

9. Klir GJ and Yuan B (1995) Fuzzy sets and fuzzy logic (Theory and applications). Prentice-Hall, International Inc.

10. Ohl igeartaigh (1982) A fuzzy transportation algorithm. Fuzzy Sets \& Fuzzy Systems. 8, 235-243.

11. Sakawa M, Nishizaki I and Uemura Y (2001) Interactive fuzzy programming for two level linear and linear fractional production and assignment problems; a case study. Eur. J. Operations Res.135, 142-157

12. Tada $M$ and Ishii $H$ (1996) An integer fuzzy transportation problem. Comput. Math. 31, 71-87.

13. Wang X (1987) Fuzzy optimal assignment problem. Fuzzy Math. 3, 101-108.
Vol. 5 No. 4 (Apr 2012)

ISSN: 0974- 6846

14. Yager RR (1981) A procedure for ordering fuzzy subsets of the unit interval. Info. Sci. 24, 143-161

15. Zadeh LA (1965) Fuzzy sets. Information \& Control. 8, 338-353.

16. Zadeh LA (1975) (1976) The concept of a linguistic variable and its application to approximate reasoning. Information Sciences, part 1,2 \& 3. Vol.8, pp:199-249; Vol.9, pp:43-58. 